\title{
Enfants et pollutions : co-conception d'un programme de sensibilisation
}

\section{Clara Boutet}

\section{(2) OpenEdition \\ 12 Journals}

Édition électronique

URL : http://journals.openedition.org/ere/5972

DOI : 10.4000/ere.5972

ISSN : 2561-2271

Éditeur

Centr'ERE

Référence électronique

Clara Boutet, «Enfants et pollutions : co-conception d'un programme de sensibilisation », Éducation relative à l'environnement [En ligne], Volume 15 - 2 | 2020, mis en ligne le 15 novembre 2020, consulté le 23 février 2021. URL : http://journals.openedition.org/ere/5972; DOI : https://doi.org/10.4000/ere. 5972

Ce document a été généré automatiquement le 23 février 2021. 


\title{
Enfants et pollutions : co- conception d'un programme de sensibilisation
}

\author{
Clara Boutet
}

1 En 2019, la jeunesse s'est mobilisée pour le climat et les plus jeunes ont pu prendre part aux manifestations du vendredi qui, pendant plusieurs mois, se sont déroulées dans les grandes villes du monde. Enfant ou adulte, nul ne peut aujourd'hui ignorer les problématiques liées à l'environnement et l'impact de l'action humaine sur leur évolution. Il est rare qu'enfants et adolescents soient tenus pour des acteurs en capacité d'agir. Or, de plus en plus, ils ont une conscience aiguë des enjeux environnementaux et de la nécessité de changer nos comportements.

Bien qu'ils soient peu sollicités pour définir ce qu'est selon eux l'écocitoyenneté et que la communauté gagnerait à ce qu'ils le soient davantage (Blanchet Cohen et Di Mambro, 2016), ils manifestent le désir de s'impliquer à travers des comportements écoresponsables. On a tendance à pointer la vulnérabilité et le manque d'expérience des enfants (James et James, 2004), mais depuis 1989, la Convention relative aux droits de l'enfant reconnaît l'intérêt de prendre en compte les considérations des enfants sur les sujets qui les concernent (art.12). En 1996, la Conférence Habitat II des Nations Unies fait d'ailleurs un pas supplémentaire en affirmant: "Les besoins des enfants et des jeunes doivent être pleinement pris en compte, notamment pour ce qui est de leur cadre de vie. Il faut prêter une attention particulière aux processus participatifs (...) et tirer parti de leurs idées, de leur imagination et de leurs réflexions sur l'environnement. » (ONU, 1996, §13).

3 La co-conception d'un produit ou d'un service par exemple, lorsqu'elle est mise en œuvre avec rigueur aux diverses étapes de l'élaboration de celui-ci, de son idéation à sa diffusion, dépasse de loin la simple consultation de l'usager. Il s'agit d'une approche participative de plus en plus plébiscitée, notamment dans le domaine de la santé publique (Massé, 2003). Cependant, à notre connaissance, la démarche de co-conception 
avec les usagers décrite ici ne compte pas de recension dans le champ de la prévention auprès de cette population particulière que constituent les enfants.

Dans le contexte actuel, l'éducation relative à l'environnement est devenue indispensable, mais pour passer du savoir à l'agir, il est nécessaire que le public visé se sente concerné en s'appropriant les concepts. Dans le but d'optimiser l'efficacité d'un programme de sensibilisation, une approche participative, associant les compétences profanes à celles d'experts, apparaît la plus apte à faire en sorte que les usagers soient également acteurs et co-auteurs de ce programme. Cela nécessite de définir les objectifs selon les désirs et la capacité d'agir des acteurs, et non en termes de prescription.

\section{Un contexte spécifique}

Face aux limites identifiées des campagnes de prévention en matière de santé et la difficulté de passer du savoir au faire, le Fonds pour l'éducation et la prévention en santé $^{1}$, a engagé son premier programme destiné aux enfants de 9 à 11 ans sur le thème des pollutions en lien avec le fonctionnement du corps. Conscients que l'usager est le plus apte à cerner ses besoins et à juger si un produit ou un service lui convient, les membres du conseil scientifique (CS) du Fonds ont décidé d'opérer une forme de décentrement pour enrichir leur regard d'expert en prenant en compte les représentations des enfants. Ils souhaitent ainsi leur donner la parole pour qu'euxmêmes puissent définir leurs attentes et besoins, et délimiter les contours (support et contenu) du programme le mieux adapté pour les sensibiliser sur ce thème.

Les questionnements relatifs à l'environnement, à travers le thème des pollutions, peuvent être considérés comme des questions "socialement vives ${ }^{2}$ ", c'est-à-dire qui font l'objet de controverses socio-scientifiques (Albe 2008; Albe et Gombert, 2010) et sont susceptibles de mettre en tension le rapport des individus à la science (Legardez et Simonneaux, 2006).

7 Pour sa première mission, le Fonds a décidé de s'adresser aux enfants afin de mieux cerner leur préoccupation de santé et identifier les connaissances qu'ils souhaitent acquérir tout en mettant en avant celles qu'ils maîtrisent. Après s'être adressé à un panel de 1000 enfants âgés de 6 à 14 ans par le biais d'une enquête quantitative de l'Institut français d'opinion publique, il est ressorti qu'en plus du thème de la pollution, en matière de santé, les enfants du panel souhaitaient « connaître les organes de [leur] corps et apprendre comment ils fonctionnent ». Ce large panel a permis de déterminer la cible du programme envisagé et d'opter pour l'âge pivot correspondant à la fin de l'école primaire, à savoir la tranche d'âge des 9 à 11 ans.

\section{Une problématique méthodologique}

8 La co-conception relève avant tout de la construction collective d'un objet autour de valeurs partagées, à travers la mise en relation de parties hétérogènes (Routier, D’Arripe et Soyez, 2017). Sa mise en œuvre dépasse l'approche participative qui se propose de joindre les publics concernés aux processus décisionnels: ici, il s'agit d'intégrer les usagers pour en faire des acteurs à part entière.

9 La co-conception ici développée s'inspire du modèle des Living Labs en Santé et Autonomie (Berthou, 2019 ; Berthou et Picard, 2017). Les Living Labs sont fondés sur le 
modèle de la startup ; ils mettent en avant un fort degré d'innovation et proposent, en matière de santé, une réflexion sur l'autonomie du patient à partir de la modification des enjeux contemporains de la santé. Ils constituent une réponse au système institutionnel de la santé et reconnaissent la nécessité de s'extraire du fonctionnement en silo, par la réunion des partenaires afin d'accélérer le processus d'innovation. Dans leurs principes, les Living Labs confèrent une égalité de voix à tous les participants, usagers compris, mais on note que leur évolution amène la plupart d'entre eux à donner une place prépondérante aux financeurs et industriels, créant de fait un déséquilibre aux dépens des usagers.

Cet engagement envers un mode de participation qui dépasse la simple concertation et consultation (Akrich, 2013), mais convie les usagers à la table, aux côtés des décideurs et financeurs, induit l'exigence de rester fidèle à une méthodologie qui se construit en même temps que son objet, c'est-à-dire selon les besoins des usagers et la nature de ces derniers. Il s'agit d'une méthodologie sui generis impliquant la conception d'outils ad hoc.

Des études déjà menées (Gutierrez, Lammel et Meunier, 2016, p. 309) encouragent d'autres recherches pour apporter des éléments de réponse à leur interrogation finale : « comment impliquer les enfants dans les actions à long terme?». Quelle forme un tel engagement prend-il lorsque les usagers concernés sont des enfants? Comment ne pas rompre l'engagement de rester fidèle à leurs attentes et besoins ?

\section{Quels enfants, quel territoire?}

Cela commence par la nécessité d'opérer un décentrement et d'accepter de se laisser surprendre: la sensibilité des enfants n'est pas construite comme celle des adultes; leur accès à la sensibilisation pourra donc emprunter d'autres chemins.

C'est sur ce véritable itinéraire méthodologique que cet article se propose de revenir. Les initiateurs du programme ont certes souhaité donner la parole aux enfants - mais sur quels critères? Et comment être sûr que les résultats obtenus par la consultation d'un échantillon d'enfants soient représentatifs de la population des enfants de 9 à11 ans présents aujourd'hui sur l'ensemble du territoire français? On suppose que selon leur milieu de vie respectif, ils ne sont pas confrontés aux mêmes problèmes liés à la pollution (Gutierrez, Lammel et Meunier 2016). De même, selon leur niveau d'éducation, leur milieu d'appartenance, ils ne partagent pas une conscience unanime de ce que recouvre le terme "pollutions", ce qui implique d'accéder à leurs représentations.

Alors comment s'assurer que le programme s'adapte aussi bien à des enfants très au fait des enjeux comme à ceux chez qui des confusions demeurent encore vives? Comment faire un programme inclusif, qui ne laisse personne de côté quand on sait que vouloir s'adresser à tous c'est souvent manquer sa cible?

Le choix d'une telle méthodologie représente un défi dont l'enjeu majeur est de n'y point déroger. Il s'agit de mettre à disposition des enfants concernés des savoirs dans lesquels ils peuvent puiser à leur gré afin, à terme, de choisir eux-mêmes les comportements adaptés en matière de préservation de l'environnement et de la santé, tel que cela est proposé par les pédagogies critiques (Illich 1970 ; Freire 1996; De Cock et Pereira 2019; Pereira 2019), répondant en cela à l'approche mutualiste qui préfère le 
développement $\mathrm{du}$ sens éthique à l'inculcation des valeurs et qui privilégie la transmission bottom-up.

\section{Éléments d'un cadre théorique} bien chez les animaux et les plantes que chez les humains. Cette difficulté peut venir du fait que la pollution est un processus biochimique extrêmement complexe » (Guillén Gutierrez, Lammel et Meunier, 2016). D'autre part, dans le champ perceptuel de la santé du point de vue de l'enfant, Jutras et Bisson (1994) notent que le « bien-être des enfants est un enjeu social important (...) cependant, les modèles sous-jacents reposent essentiellement sur une perspective individuelle et sur une vision adulte de ce qu'est la santé." Les auteurs adoptent comme postulat l'idée "qu'en comprenant mieux comment les enfants pensent la santé, on pourra identifier des méthodes plus appropriées à chaque âge pour changer les connaissances, les attitudes et les comportements ». C'est précisément le point de départ de l'équipe en charge de l'élaboration du programme de prévention santé dont il est ici question. D'où l'intérêt d'une approche participative rigoureuse et intégrale telle que le prévoit le processus de co-conception.

On parle de co-construction «des dispositifs et de leurs utilisateurs pour marquer l'intrication entre ces termes et le fait qu'à une vision linéaire de l'innovation qui dissocierait l'élaboration technique de l'appropriation sociale, on substitue une série de configurations en évolution qui travaillent l'articulation entre technique et usage " (Akrich, 2013). Cela justifie de faire appel à une designer de services pour initier le programme à partir de la pensée design. Un designer de services applique les méthodes du designer à la résolution de problèmes complexes, en faisant appel à la pensée créative comme à la pensée analytique, mettant ainsi en œuvre des processus à la fois artistiques et scientifiques (Lietdka, 2015). Il est, en somme, un résolveur qui s'immerge dans son objet d'étude et l'écosystème qui l'accompagne (Pruneau et coll., 2017).

L'approche participative de la co-conception qui place l'usager et ses besoins au centre de ses préoccupations vient renforcer les axes chers au développement et à la mise en pratique de l'éducation relative à l'environnement (ERE), d'après les grands principes de la pédagogie environnementale dégagés par Lucie Sauvé (1997). Pour rappel, l'ERE a pour objet les relations entre personne(s)-société(s)-environnement(s); elle préconise une approche globale et systémique tant de la personne que de l'environnement (à travers le concept d'écosociosystème). Cette approche permet de sortir de la pensée en silo et d'accéder à la pensée complexe (Sauvé, 1997).

De plus, elle s'efforce de mettre en lumière le caractère contextualisé, complexe, relatif et incertain de toute problématique, en adoptant une pédagogie de terrain et en abordant l'environnement à travers le milieu de vie quotidien. L'évocation de situations vécues constitue l'une des conditions majeures pour que les enfants expriment la richesse de leurs représentations et qu'ils se perçoivent comme des acteurs environnementaux (Blanchet Cohen et Di Mambro, 2016).

Par ailleurs, l'approche dont il est ici question s'appuie sur des méthodes actives, impliquant l'apprenant et développant l'esprit critique. Elle s'emploie à favoriser l'engagement actif des apprenants dans la gestion de situation d'apprentissage et la construction du savoir. Elle contribue à la mise en avant du travail coopératif au sein

Éducation relative à l'environnement, Volume 15 - 2| 2020 
d'une même communauté. Cela passe par la recherche d'informations dans des ressources diverses, la confrontation à des sources scientifiques avec explication de la démarche, la vérification de l'information ou encore la conscientisation du caractère non figé de la notion de vérité scientifique.

Si l'approche ici retenue s'est attachée à prendre en compte les représentations des enfants, elle ne l'a pas fait à partir d'une étude systématique des représentations telle que d'autres l'ont pratiquée en France, comme Serge Moscovici (1976) du côté de la psychologie sociale et Claudine Herzlich en sociologie de la santé $(1969 ; 1972)$.

Depuis, les travaux sur les représentations de la santé des adultes sont nombreux (D'Houtaud, 1976; Guyon, 1990), mais plus rares sont ceux qui concernent celles des enfants (Garnier, Marinacci et Quesnel, 2007, sur celles de la maladie et de l'alimentation). L'étude menée par Jutras et Bisson (1994) constitue une base importante en ce qu'elle prend en compte les interactions des enfants au sein de leur écosystème pour améliorer leur bien-être, en accordant une importance cruciale à la prévention (Pransky, 1991).

Concernant les représentations sociales liées aux pollutions et leur association à la notion de saleté (Pruneau et coll., 2005), on peut distinguer d'une part, les différents types de pollutions et d'autre part, leurs caractéristiques à partir de plusieurs critères : visible/invisible; proche/lointaine; immédiate/qui dure; franche/non franche (Caillaud, 2010).

\section{Démarche méthodologique}

L'approche quantitative s'est limitée à l'enquête préliminaire évoquée précédemment, afin d'affiner le choix du groupe d'âge à privilégier. Elle a permis de décrire et comprendre les motivations et inclinations des commanditaires du programme à développer. Une approche qualitative a été privilégiée lors de toute la phase exploratoire à travers des ateliers-tests menés avec des groupes d'enfants d'une part, et auprès des médiateurs d'autre part.

Les ateliers de la phase exploratoire ont impliqué 31 enfants issus de quatre groupes pour lesquels le dispositif d'interaction n'était pas tout à fait le même, bien que suivant une trame commune, réajustée entre les séances, selon le principe itératif propre à la co-conception et mis en œuvre tout au long du processus. Concernant l'âge des destinataires, la cible choisie est celle des enfants de CM2, dernier palier de l'école primaire qui précède l'entrée au collège. La tranche des 9 à11 ans a été retenue en raison de la capacité des enfants à appréhender les enjeux, amorcer une réflexion critique, tout en restant attachés à des univers imaginaires. Afin d'être inclusifs, les initiateurs du programme ont choisi de mettre en œuvre la démarche de co-conception avec des enfants représentant l'ensemble du territoire métropolitain (Paris et banlieue parisienne, et milieu rural). L'implantation au cœur du milieu scolaire semble la plus à même de créer des opportunités pour l'éco-responsabilisation des enfants (Scott, 2011) afin de s'étendre ensuite aux familles pour favoriser le développement de l'esprit critique de l'enfant (Hayward, 2012). Les centres de loisirs ont aussi été explorés pour leur complémentarité éducative avec le milieu scolaire. 


\section{La réalisation du cahier des charges}

26 À partir de la réalisation d'un cahier des charges, les grandes lignes et principales contraintes sont définies. Cet outil permet de faire un point sur les attentes, sans les figer, et de développer divers exemples (notamment les "frontières et possibles du projet"). Il s'agit donc d'un état des lieux non exhaustif évoquant la pluralité des domaines auquel le projet peut renvoyer avant qu'il ne soit véritablement défini.

La designer passe en revue plusieurs approches possibles à travers divers exemples, afin de déterminer celles qui fonctionnent le mieux chez les enfants et la raison de leur succès. Où cherche-t-on à se situer ? Un axe « réaliste/métaphorique » qui détermine la proportion de réalisme et la place qu'on laisse à l'imaginaire croise un axe allant de la transmission descendante à l'apprentissage proactif. L'étape de la réalisation du cahier des charges se veut décisive dans le cheminement de la designer afin qu'elle délimite sa zone d'expression en fonction des contraintes.

\section{Modalités de collecte des données}

Lors des séances, les deux médecins et l'ethnologue sont sur place, en observation. L'observation est réalisée à partir d'une méthode ethnographique; elle intègre les interactions entre les acteurs et se montre attentive aux formulations ou plus précisément aux "façons de dire ». Cela permet une entrée qualitative dans les représentations des acteurs. Ainsi userons-nous dans la description à suivre, de verbatim qui rendront compte autant de l'ambiance générale des ateliers que des propos des enfants, mais également des méthodes utilisées par les animatrices des ateliers, rôles investis par la sociologue qualitativiste et la designer de l'équipe. Les données ont été collectées par observation, prises de notes, enregistrements sonores voire captures vidéos (lors des séances parisiennes pour ces dernières).

Complémentaires de celles de la designer, les compétences de la sociologue qualitativiste, spécialiste du décentrement, garantissent un accès aux représentations préservé des idées préconçues en ce qu'elle fonde son expertise sur les dires de la population concernée.

\section{Description des ateliers de co-conception}

Le programme est élaboré grâce à plusieurs ateliers de co-conception dont l'objectif est de recueillir les représentations des enfants à travers des exercices projectifs visant à les mettre en situation. Ces ateliers se sont déroulés à partir d'une trame commune qui a subi des modifications d'une séance à l'autre, selon le processus itératif propre à la co-conception. Les réajustements ont permis d'accentuer les questionnements relatifs au corps. À Paris, les enfants ont été recrutés par une agence spécialisée sur leur capacité à prendre la parole et dissociés en deux groupes selon leurs activités, lectures récurrentes, abonnements à des magazines, etc. Le Groupe 1 (G1) comporte les enfants dits plus sensibilisés et le Groupe 2 (G2), les moins sensibilisés. Leurs membres n'ont pas connaissance du thème qui sera abordé et les séances se déroulent dans une salle dédiée, équipée d'une caméra, de micros et d'une vitre sans tain derrière laquelle se trouvent les observateurs. Le dispositif est différent en Aveyron (Groupes 3 et 4 ) où les 
enfants ont répondu sur la base du volontariat à venir parler du thème de la pollution ; certains d'entre eux se sont montrés particulièrement sensibilisés ${ }^{3}$.

L'idée est de proposer aux enfants un "espace de tous les possibles", un univers au sein duquel leur parole peut se libérer. On n'attend pas d'eux des «bonnes réponses »; ils ne sont pas en situation d'évaluation, au contraire, ils peuvent " dire des trucs fous " et laisser vagabonder leur imagination. La mise en place du cadre implique aussi de rappeler la règle du respect de la parole de l'autre. Cela permet d'obtenir des informations sur leurs goûts, les médias qu'ils apprécient et qui suscitent leur intérêt.

Ils se montrent concernés par les problèmes environnementaux proches de leur réalité vécue - ce qui ne signifie pas qu'ils ne sont pas conscients des problèmes globaux, qu'ils ne sont pas capables de proposer des solutions et de se projeter en tant qu'acteurs.

Dans une démarche qualitative et afin de sonder l'état des connaissances des enfants de CM2 sur les pollutions, ce qu'ils aimeraient apprendre et de quelle manière, l'équipe est allée à la rencontre de quatre groupes, deux à Paris, deux en milieu rural ainsi constitués : trois groupes de 8 enfants et un de 7 enfants (G3), composés de 17 filles et 14 garçons.

Chaque séance de co-conception est animée par la sociologue et la designer. La première a pris en charge l'animation pour sonder les enfants et recueillir leur parole ; la seconde s'est chargée des activités impliquant des outils confectionnés par ses soins (voir par exemple, la cartographie du quotidien, Fig. 1). Les séances, d'une durée de $2 \mathrm{~h} 30$, ont eu lieu sur le temps extrascolaire. L'atelier s'ouvre sur une présentation où chaque enfant échange d'abord brièvement avec son voisin, pour ensuite le présenter (prénom, âge, héros, dessin animé, livre ou jeu qu'il affectionne particulièrement). La première phase se caractérise par un échauffement créatif visant à créer un collectif et instaurer un univers de tous les possibles le temps de la séance, en imaginant quel "super-pouvoir» chacun aimerait avoir. Ensuite, on interroge les enfants sur les activités scolaires et périscolaires qui les ont particulièrement marqués.

Vient ensuite l'étape cruciale de l'exploration de la notion de pollution, d'abord par association d'idées et d'images, puis par un approfondissement illustré par une carte mentale (Tableau 1.). Si certains ont pu donner plusieurs éléments de réponse, on a veillé à ce que chacun s'exprime au moins une fois, excepté dans le G1 où les éléments ont été relevés selon le modèle du "cadavre exquis » : chacun notait le mot auquel il pensait puis repliait la feuille pour la passer à son voisin. Cela explique qu'il s'agisse davantage de mots-concepts que d'images. Ils ont ensuite pu s'exprimer sur les mots qu'ils avaient choisis après un exercice divergent qui consistait à piocher dans un panel d'images (prédécoupées et sélectionnées dans divers magazines) une illustration leur évoquant la pollution et une autre, le contraire de la pollution. 
Tableau 1 : Exploration de la notion de pollution

\begin{tabular}{|c|c|c|c|}
\hline $\begin{array}{l}\text { G1 } \\
\text { Paris } \\
+ \text { sensibilisés }\end{array}$ & $\begin{array}{l}\text { G2 } \\
\text { Paris } \\
\text { - sensibilisés }\end{array}$ & $\begin{array}{l}\text { G3 } \\
\text { Aveyron }\end{array}$ & $\begin{array}{l}\text { G4 } \\
\text { Aveyron }\end{array}$ \\
\hline $\begin{array}{l}\text { Pollution durable } \\
\text { Terre } \\
\text { Réchauffement climatique } \\
\text { Voitures } \\
\text { Déforestation } \\
\text { Planète } \\
\text { Air } \\
\text { Voitures }\end{array}$ & $\begin{array}{l}\text { Voitures } \\
\text { Saleté } \\
\text { Usines } \\
\text { Nuage noir } \\
\text { Fumée } \\
\text { Pôle Nord } \\
\text { Pollution de la terre }\end{array}$ & $\begin{array}{l}\text { Cigarette } \\
\text { Gaz } \\
\text { Gáchis } \\
\text { Déchets } \\
\text { Nucléaire } \\
\text { Otarie coincée avec une boite } \\
\text { de conserve } \\
\text { Oiseau ouvert avec des déchets } \\
\text { a l'interieur } \\
\text { Plastiques } \\
\text { Tortues qui se prennent dans } \\
\text { des filets } \\
7 \text { continent } \\
\text { Montagne de déchets en Inde } \\
\text { «On a tout gáché } \% \\
\text { L'bomme a fait beaucoup de } \\
\text { dégåts. }\end{array}$ & $\begin{array}{l}\text { Fumée et voitures en Chine } \\
\text { Usine trés schématisée (vue } \\
\text { dans un livre) } \\
\text { Usine qui rejette de la fumée } \\
\text { Fonte des glaces } \\
\text { Déchets } \\
\text { \& Contre-usine * - C'est } \\
\text { quoi ? ca existe pas mais un } \\
\text { truc contre les usines } \\
\text { Bćbés ours polaires } \\
\text { Disparition des animaux, ils } \\
\text { ont plus d'environnement, } \\
\text { ils mangent du plastique } \\
\text { Véritable danger } \\
\text { Fin du monde progressive } \\
\text { On coupe les arbres : } \\
\text { deforestation }\end{array}$ \\
\hline
\end{tabular}

\section{Mise au jour des biais}

À Paris, une note explicative adressée aux parents a circulé avant l'atelier. La mention du thème "pollution» sur la note tend à relativiser la spontanéité de certaines réponses lorsqu'en début de séance, les enfants ont répondu que s'ils avaient un superpouvoir, ils aimeraient se téléporter " pour voir qu'est-ce que peut devenir la planète avec la pollution qu'on lui inflige » (G1) ou avoir le «pouvoir de l'anti-pollution en claquant des doigts » (G2).

Pour recueillir un maximum d'informations sur leurs représentations de la pollution, la mise en place du cadre est décisive. Contrairement aux séances parisiennes, les séances aveyronnaises se déroulent dans un lieu familier des enfants: pour le groupe 3, les enfants sont issus d'une même classe. Quant au groupe 4, la séance se déroule dans une des salles de l'école, avec des enfants issus de plusieurs classes : deux sont en CM1 et le frère de l'un d'eux, élève de $6^{e}$ est présent. Bien que cela n'ait pas semblé perturber la séance, il est important de noter que pour ce groupe, la directrice de l'école est restée dans la salle, en retrait.

\section{Suite du déroulement des séances}

Une autre activité consiste à placer la pollution dans divers lieux du quotidien : la maison, le jardin, l'école, la rue... L'enfant est ici amené à se projeter dans son quotidien et un environnement qui lui est familier, plus que sur des questions d'environnement global (qu'il a tendance à citer plus spontanément). 


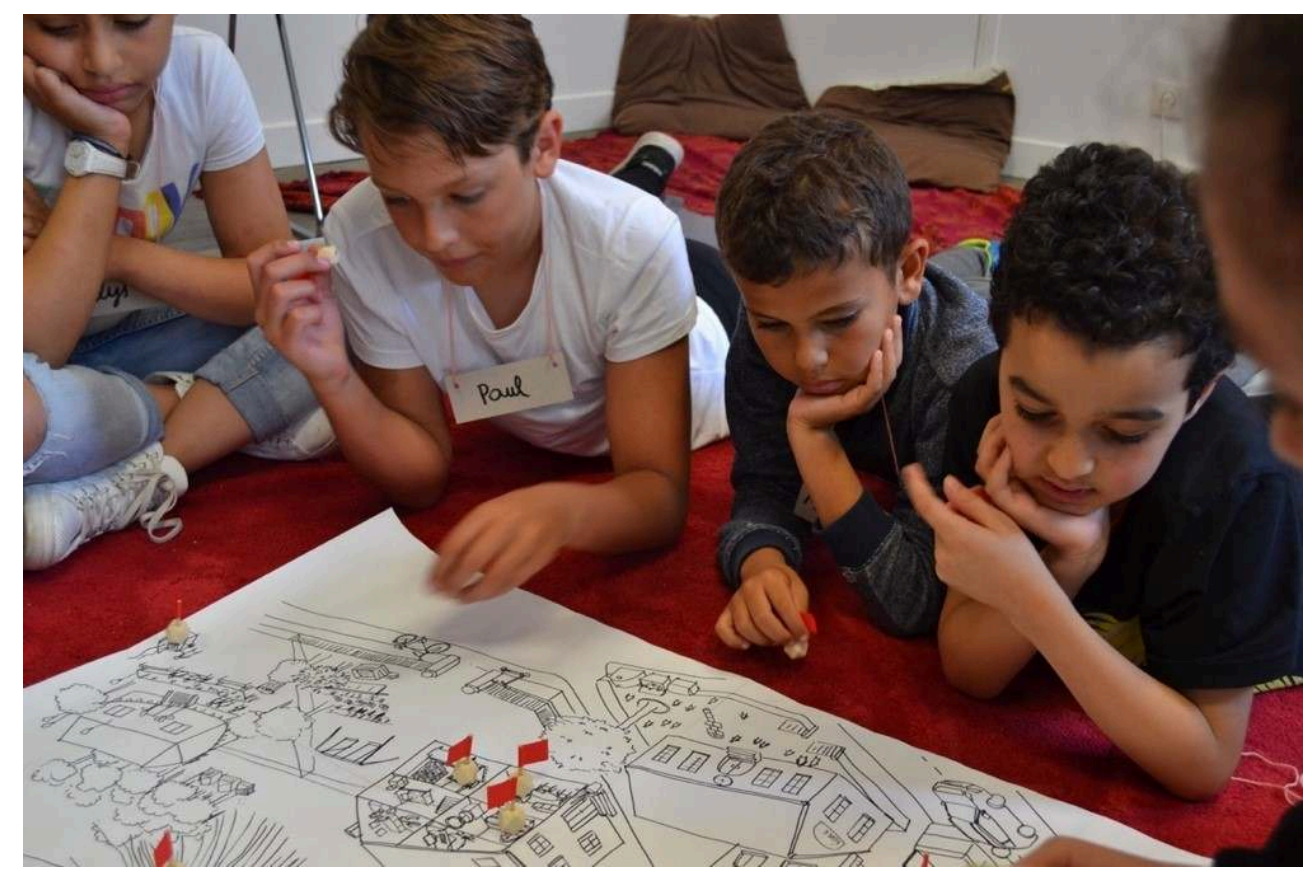

Figure 1 : Cartographie du quotidien (G2), juin 2019

(c)Fanny Prudhomme pour le Fonds pour l'éducation et la prévention en santé

Le jeu du "ça pollue / ça ne pollue pas » a permis d'évaluer l'accès à la pensée complexe des enfants, notamment grâce à la possibilité de placer l'élément dans une section tierce $d u$ "ça dépend». Cette activité ludique explore différents types de pollution En piochant, par exemple, un e-mail: «ça pollue car ça envoie des ondes mais c'est mieux qu'utiliser du papier - donc ça dépend» (G2); ou en piochant le dentifrice : «produits chimiques, ça pollue le corps, mais pas que, y a aussi l'emballage que l'on jette » (G1); pour certains, c'est même "cancérigène ", «la pâte, faut pas l'avaler, c'est du plastique » (G3).

Dans cet atelier, nous regardons le point de vue des enfants afin de déterminer : «Ce qu'on aimerait comprendre ? Ce qu'il faut savoir ? À qui je pourrais poser la question ? Comment?» (Fig.2). On interroge les enfants sur ce qu'ils auraient envie de savoir et quel support, selon eux, serait le mieux adapté. L'objectif étant, du point de vue des enfants, d'obtenir « une action que je peux mener, moi, dans mon espace » et du point de vue de l'équipe, d'imaginer le contenu pédagogique adéquat à partir du recueil de ces conceptions.

\section{Résultats}

\section{La pollution se pense au pluriel}

Cette phase d'exploration a permis de constater que pour ces enfants, plusieurs types de pollution nous entourent: pétrole, pollution liée aux transports (train, voiture, moto), déchets, plastique, pollution de la mer, etc. Aussi, la pollution alimentaire (pesticides), «mentale " (par les écrans) et, en milieu rural, les pollutions lumineuse («On voit la lumière, on ne voit plus les étoiles»-G3), spatiale et publicitaire ont été mentionnées. Le G2, issu de la région parisienne et moins sensibilisé, a plus de mal à 
définir les «types» de pollution. Les enfants reprennent, par exemple, «fumée, pétrole » et reviennent beaucoup à la pollution de l'air.

- La pollution de l'air concerne les éléments sensoriels. En voici quelques exemples: sur l'odorat, «ça sent le gaz » en voiture (G3) ou lorsqu'on repeint une pièce, «c'est pas bon pour les poumons » (G2). On se sent attiré par l'odeur, mais on sait qu'elle est nocive (souvent parce qu'un parent nous le signifie et/ou parce qu'on fait l'expérience d'un mal de tête...). De même : «J'ai remarqué, quand je sens un parfum, j'ai un truc chimique sur ma langue - comme si j'avais le goût dans ma bouche - j'aimerais savoir pourquoi » (G2).

- La « pollution sonore" » à travers le bruit des voitures : « Ça fait mal aux oreilles» (G3) ; « Si tu vas dans la campagne, t'entends les oiseaux, la nature, alors que si tu vas dans une grande ville, t'entends des industries, on se croit dans un autre monde. Ça fait mal aux oreilles.» (G4).

- La pollution visuelle s'illustre principalement par les déchets accumulés à travers des éléments du quotidien comme les «palettes Mc Do » (G3), mais surtout des images choc de montagnes de déchets vues à la télévision ou dans des magazines. Dans la mer également, où une enfant du G3 se souvient de «tout petits plastiques en décomposition» dans ses cheveux.

Ainsi la pollution apparait-elle comme une nuisance qui agit sur l'un des sens voire plusieurs. Elle est perçue à la fois comme une gêne qui a un impact sur les organes sensoriels et comme une souillure (Douglas, 2001). Elle est un élément qui peut nous toucher par l'air ("La pollution, ça pollue l'air et l'air après ça se diffuse partout. », G1), par ce qu'on mange, ou dans la mer « mais ça nous touche moins parce qu'on n'y va pas tous les jours ", G2).

\section{Types d'apprentissage souhaités}

La réalisation d'une carte mentale permet de visualiser les associations d'idées. Les enfants n'ont pas l'habitude qu'on leur pose des questions sur des choses qu'ils n'ont pas apprises au préalable. Il faut les aider à développer leur pensée : "Où est la pollution? Tu es proche physiquement de l'espace de pollution? Comment tu le sais?» afin d'obtenir des indices sur les sources d'information des enfants (Fig.2).

À partir des grands axes de la carte, on peut identifier quelques thématiques : tout ce qui rentre dans le corps et est lié à son fonctionnement ce qui est présent dans l'air, les objets qui monopolisent l'attention, les nuisances. On se soucie de prendre aussi en compte ce qu'ils peuvent imaginer d'insolite afin de ne pas opérer de restrictions selon une logique de raisonnement d'adulte.

Les boucles itératives sont réalisées sur la base des éléments recueillis auprès de G1 et G2. Les groupes $\mathrm{G} 3$ et $\mathrm{G} 4$ ont permis de conclure que les enfants souhaitaient apprendre dans des lieux de savoirs (école, laboratoire, bibliothèque...), en famille, avec leurs pairs, rarement seul, en faisant des expériences, en menant l'enquête, en rencontrant des personnes spécialistes qui expliquent, et sur une multiplicité de supports (Fig.2).

On relève aussi la possibilité de croiser des univers (par exemple, un théâtre dans un laboratoire), ou des associations qui nous paraissent inusitées (par exemple, la volonté d'apprendre dans un laboratoire, avec un journal, en faisant du sport), soulignant la capacité des acteurs à rompre les frontières rationnelles souvent imposées par les adultes. 

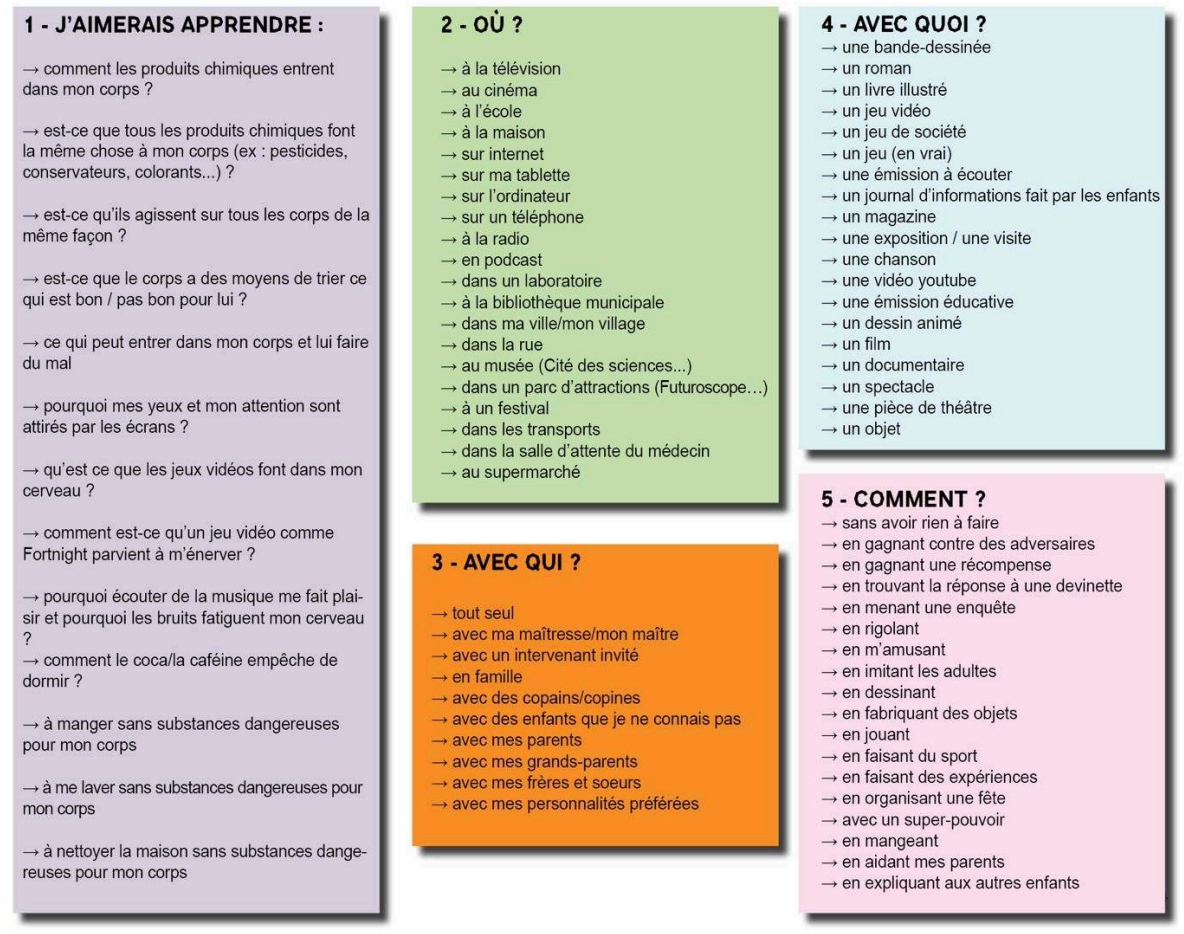

Figure 2 : Liste "J'aimerais apprendre" (G3 et G4)

(C)Fanny Prudhomme pour le Fonds pour l'éducation et la prévention en santé

\section{Bilan des séances}

47 La séance est une mise en situation d'interaction sociale menée par la sociologue qui souligne certains éléments ou en filtre d'autres afin de garantir la fluidité des échanges et la liberté de parole des enfants. Ceux-ci ne doivent pas réellement comprendre l'objectif de l'exercice, afin d'éviter une situation dans laquelle ils chercheraient à répondre du mieux possible ou donner la « bonne réponse ».

Dans le cadre institué, spontanément les enfants étaient attentifs aux consignes et s'écoutaient entre eux. On note un peu plus de chahut en G3, mais cela s'explique par le fait que les enfants appartiennent à la même classe et qu'ils partagent des références communes, ont l'habitude de jouer les uns chez les autres, se prêtent des livres, sont partis en voyage scolaire ensemble... Aussi, ils n'étaient pas en présence d'un adulte référent de leur environnement ${ }^{5}$.

49 Finalement, les enfants se sont montrés informés, conscients des de la complexité et des enjeux liés à l'environnement, lucides quant aux ficelles du monde dans lequel ils vivent. Par exemple, concernant l'incitation à ne pas gaspiller à la cantine, « on n'est pas dupes ", le surplus finit par être jeté !. Les enfants ont conscience d'une sorte de connexion entre le corps et l'extérieur, mais ignorent comment elle se fait (ce qui, de ce point de vue, ne les différencie pas des adultes).

50 Les enfants de G3 et G4 montrent un sens pratique plus développé, ainsi qu'un sens du concret et un attachement au contact avec la nature, ce qui s'explique par leur environnement quotidien - poules, compost, étoiles, etc. Ils se sont montrés très 
marqués par les images concernant les atteintes à l'environnement et particulièrement, aux animaux. On relève, contrairement aux séances parisiennes, le contact avec la nature, le vivant, mais aussi la mort (par exemple, à travers les cadavres d'animaux pour nourrir le milan royal lors d'une sortie à la déchetterie). On peut associer le sens pratique associé au thème de la mort (par exemple : «Si ça tue les insectes, ça tue aussi notre organisme »; « On est tous morts si les abeilles disparaissent, il n'y aura plus de fruits, plus de plantes ", G4). De même, ils lient l'effort physique et le fait de faire par soi-même, comme des facteurs minimisant la pollution : toutes les énergies ne sont pas polluantes, comme celle que produit mon corps lorsque l'on pédale: "On produit de l'énergie, mais ça pollue pas. Elle est dans notre corps. Notre corps, quand il fabrique de l'énergie, il fabrique aussi un moyen de la recycler » (G4).

\section{Pensée complexe et représentations en lien avec le corps}

51 Selon les enfants, des choses « rentrent dans le corps » et nous font du bien, d'autres non. On trouve l'idée de flux qui entrent et sortent. Les vêtements ou la peau (que l'expression "barrière cutanée " illustre si bien) renvoient à l'idée de limites et frontières. Le corps est perçu comme une enveloppe perméable (peau, nez, bouche, oreilles, cerveau) sur laquelle les éléments extérieurs ont des effets, à travers ce qu'on mange, ce qu'on entend, ce qu'on touche par exemple.

Aussi, les enfants se montrent conscients que les conséquences d'un acte peuvent être différées dans le temps et dans l'espace du moment de sa réalisation. Par exemple, l'emballage plastique jeté et ingéré par un animal peut entrer ainsi dans la chaîne alimentaire et, à terme, affecter la santé humaine. Les enfants ont également conscience qu'un impact délétère peut être invisible de prime abord.

Néanmoins, il demeure des zones d'inconnues, en particulier autour des artefacts capables de " polluer le cerveau et l'esprit » (G3 et G4), avec des objets qui prennent le contrôle de nos vies (les écrans, la lumière bleue, des ondes, etc.).

Enfin, on note chez les enfants leur goût prononcé en faveur des formules choc (les sodas, «c'est du cancer à boire »). On souligne aussi leur intérêt pour l'humour, pour s'amuser, se mettre dans la peau d'un autre ; ils aiment avoir la liberté de «faire des bêtises». Combiné, cela peut donner un humour noir, grinçant. Aussi, les enfants aiment-ils jouer à se faire peur et ne sont pas enclins à traiter les sujets sérieux (tels que la maladie ou la mort) de façon sérieuse. Ils neutralisent d'eux-mêmes le côté moral et très anxiogène du thème de la pollution et sont attirés par le côté fascinant des « super pouvoirs » de la nature et du corps. Ils se montrent prêts à s'engager comme à mobiliser leur entourage.

\section{Les enfants, acteurs de l'environnement}

Parmi les savoirs que l'on cherche à mettre à la disposition des enfants, on fait en sorte qu'ils puissent répondre aux questions qu'ils se posent, par exemple sur l'origine d'un polluant : tel polluant existait-il dans le passé ? Existera-t-il dans le futur ? D'où vientil ? Ces questions sont croisées avec celles qui ont trait au fonctionnement du corps : Un polluant qui est entré dans mon corps va-t-il y rester pour toujours? Combien de temps passe un polluant dans mon corps? Y a-t-il des endroits dans mon corps où le polluant reste plus longtemps? Est-ce qu'il est possible d'éliminer un polluant, de «nettoyer » 
mon corps ? Est-ce qu'un polluant peut être éliminé par le corps, mais avoir quand même des effets résiduels sur la santé ? Par quel biais un polluant peut-il entrer/sortir de mon corps? Etc.

De là, la difficulté est double : d'une part, il faut expliquer le fonctionnement du corps humain, des différents organes et différents systèmes (respiratoire, digestif, neurocognitif, etc.), en fonction de ce qui est recevable pour un enfant de cet âge et du programme scolaire (ne pas faire doublon, ni anticiper l'année suivante); d'autre part, on doit considérer l'état de la science sur ces questions (par exemple, sur les dangers réels provoqués par l'ingestion de nanoparticules de plastiques et ce que cela provoque sur les reins, ou encore la nocivité des pesticides).

57 Ces apprentissages prendront la forme d'une "frise du savoir » complétée par les enfants à partir de recherches effectuées dans des ressources multiples. La fabrication d'objets de médiation les enjoint ensuite à donner forme à ces connaissances en mettant en œuvre leur créativité. Ces objets seront ensuite ramenés à la maison ${ }^{6}$ de façon à ce que la médiation se poursuive au sein du foyer.

In fine, il s'agit de proposer un ensemble de gestes qui, à terme, ont un impact positif sur la santé (sa propre santé, celle de son entourage, mais aussi de façon plus générale sur la santé des populations). Ces gestes, nouvelles habitudes ou comportements, portent par exemple, sur les modes de transport, la consommation des produits (regarder la provenance des produits, éviter certaines marques, privilégier les produits faits maison, emprunter ou louer au lieu d'acheter neuf et donner ce dont on n'a plus besoin au lieu de jeter, etc.), jusqu'au choix de métiers, avec une reconsidération du « faire » à partir de métiers qui ne participent pas à la dégradation de l'environnement.

Si le thème choisi pour ce premier programme porte bien sur des questions environnementales impliquant un positionnement écologique, les préoccupations de ses initiateurs ne sont pas en premier lieu liées à l'environnement, mais bien à l'éducation et la prévention en santé. Or, dans le contexte actuel, et d'après ce que les enfants ont pointé comme étant leur préoccupation première, l'impact des pollutions apparaît comme un thème incontournable.

Cette approche participative consiste en une analyse de besoins adaptée aux enfants. Par-delà l'enjeu ludique et pédagogique, il s'agit d'accéder à des actions durables et réalisées en conscience: on touche ici le délicat objectif d'un programme de sensibilisation et la difficulté d'en évaluer les effets. Seule une étude d'impact dira si la méthode de co-conception aura tenu ses promesses en donnant envie aux enfants de participer à des actions concrètes pour prendre soin de leur corps et de leur environnement.

61 Dans sa conception, le programme est compatible avec les trois finalités de l'éducation relative à l'environnement: 1) la participation à la préservation de la qualité de l'environnement, 2) dans l'épanouissement de la personne quant à sa relation à l'environnement (pour garantir son bien-être, et donc sa santé), 3) à partir de principes pédagogiques adaptés, favorisant la pensée autonome, critique et collaborative (Sauvé, 2009). 


\section{Perspectives}

La phase d'exploration que nous avons présentée visait à recueillir des informations quant aux intérêts des enfants, leur niveau d'information et leurs représentations « des pollutions ", insistant sur le fait qu'il en existe plusieurs types, tels qu'ils l'ont euxmêmes signalé. Ensuite, s'est ouverte la phase dite «de test » durant laquelle il était question de mettre les enfants en situation afin d'ajuster le programme. Ce processus itératif est mené jusqu'à la fabrication du prototype d'un parcours éducatif et sa diffusion finale. Ainsi le programme est-il appelé à évoluer jusqu'à sa mise en circulation.

63 À partir des données recueillies lors des ateliers de co-conception que nous avons décrits, la designer a proposé une première version du programme. Celle-ci croise trois approches $^{7}$ (scientifique, journalistique et artistique) afin de répondre au souhait des enfants et de les inviter à enquêter, tout en associant l'objectif pédagogique d'acquérir de nouvelles connaissances. Les trois modules du programme proposent d'associer le savoir au faire à partir d'abord d'un « labo » où s'informer et expérimenter, ensuite par la fabrication de supports de médiation pour transmettre ce qu'on a compris ou appris à ses pairs ou son entourage et enfin, par l'organisation d'un événement (exposition ou spectacle par exemple) de plus ou moins grande ampleur.

64 Aussi, on retrouve la volonté de mettre en œuvre la créativité et l'imagination des enfants en leur permettant de confectionner des outils de médiation qui serviront à cette transmission. Le tout doit être à géométrie variable, selon le nombre d'élèves dans une classe, selon le temps que l'enseignant.e ou l'animateur.trice souhaite consacrer au programme et la possibilité de puiser un élément ou l'autre sans suivre le déroulement de bout en bout. Il s'agit autant de favoriser les connaissances que les compétences de l'enfant, en imbriquant les unes aux autres. A noter enfin que selon nos observations, les enfants des groupes à l'étude souhaitent une approche mêlant le rire au trash, sur un support multimédia, c'est-à-dire à partir de plusieurs modes d'expression.

65 Dans la poursuite de l'élaboration du programme, sept thématiques impliquant un polluant et son impact sur le corps ont été déterminées à partir de l'exploration menée auprès des enfants. Par exemple, la première entrée choisie concerne l'impact des emballages plastiques sur l'environnement et la santé. Cette entrée nécessite une approche systémique et globale, depuis les ressources jusqu'à l'humain, en considérant son corps et sa santé.

Il s'agit de respecter un idéal mutualiste selon lequel les actions sont relatives à des choix, en connaissance de cause. Le rôle des experts en prévention est d'abord de mettre les savoirs à dispositions et non d'imposer des comportements. Ceux-ci doivent découler de choix posés comme libres. Ce parti pris rejoint un véritable idéal politique où l'autonomie des acteurs prime, ce qui induit également un choix d'autonomie dans la diffusion du programme : c'est-à-dire que l'objectif n'est pas de le faire entrer dans les programmes scolaires obligatoires. Il s'agit plutôt de proposer et d'offrir des suggestions, des savoirs, des solutions, des outils dans lesquels on peut puiser librement.

Cette étude met en évidence d'une part, l'intérêt d'associer les sciences sociales et une approche qualitative pour le développement d'un programme de prévention en santé et d'autre part, l'importance d'adopter à cet effet une méthode rigoureuse pour ne pas se 
détacher de la réalité des enfants. Nous avons pu confirmer la pertinence d'une démarche de co-conception en matière de prévention et d'éducation relative à l'environnement en vue de favoriser l'acquisition de savoirs qui, une fois intégrés, pourront avoir un effet sur les comportements.

\section{BIBLIOGRAPHIE}

Abric, J.-C. (dir.) (1994). Pratiques sociales et représentations. Paris : PUF.

Akrich, M. (2013). Co-construction. Dans Casillo, I. avec Barbier, R., Blondiaux, L., Chateauraynaud et coll., Dictionnaire critique et interdisciplinaire de la participation. Paris : GIS Démocratie et Participation. Récupéré sur www.dicopart.fr/fr/dico/co-construction .

Albe, V. (2008). Pour une éducation aux sciences citoyenne : Une analyse sociale et épistémologique des controverses sur les changements climatiques. Aster, 46, 45-70.

Albe, V. (2011). Changements climatiques à l'école : Pour une éducation sociopolitique aux sciences et à l'environnement. Éducation relative à l'environnement, 9.

Albe, V. et Gombert, M.-J. (2010). Intégration scolaire d'une controverse socioscientifique contemporaine : savoirs et pratiques d'élèves pour appréhender les savoirs et pratiques de scientifiques. Recherches en didactiques des sciences et des technologies, 2.

Berthou, V., (2019). De l'intention d'innovation à son institutionnalisation. Le cas des Living Labs en Santé \& Autonomie. Cahiers COSTECH, 2. Récupéré sur www.costech.utc.fr/CahiersCOSTECH/

Berthou, V. et Picard, R. (2017). «Les Living Labs », ces leviers d'innovation en santé publique », F.F.E., Annales des Mines - Réalités industrielles, (2), 68-72.

Blanchet Cohen, N., et Di Mambro, G., (2016). L'écocitoyenneté chez les enfants : potentiel et paradoxe. Éducation relative à l'environnement, 13(2. Récupéré sur https://id.erudit.org/iderudit/ 1052535ar

Buchanan, R. (2001). Human dignity and human rights : Thoughts on the principles of humancentered design. Design Issues, 17 (3), 35-39.

Caillaud, S. (2010). Représentations sociales et significations des pratiques écologiques : Perspectives de recherche, Vertigo, (10) 2. Récupéré sur https://id.erudit.org/iderudit/045522ar De Cock, L. et Pereira, I. (2019). Les Pédagogies critiques. Paris : Agone.

Douglas, M. (2001). De la souillure. Essai sur les notions de pollution et de tabou. Paris : La Découverte. D’Houtaud, A. (1978). Les représentations de la santé, recherche dans un centre de bilan de santé en Lorraine. Revue internationale d'éducation pour la santé, 19 (2).

Freire, P. (1996). Pédagogie de l'autonomie. Toulouse : Erès.

Garnier, C., Marinacci, L. et Quesnel, M. (2007). Les représentations sociales de l'alimentation, de la santé et de la maladie des jeunes enfants. Service social, 53 (1), 109-122.

Guillén Gutierrez E., Lammel, A. et Meunier, J.-M. (2016). Les enfants face aux menaces environnementales : la représentation de la pollution. Enfance, 3 (3), 299-313. 
Guyon, L. (1990). Quand les femmes parlent de leur santé. Québec : Les Publications du Québec.

Hayward, B. (2012). Children, Citizenship and Environment. Nurturing a Democratic Imagination in a Changing World. Routledge : London.

Herzlich, C. (1969). Santé et maladie. Analyse d'une représentation sociale. Paris : EHESS.

Herzlich, C. (1972). La représentation sociale. Dans Moscovici, S. (éd.), Introduction à la psychologie sociale. Paris : Larousse, Sciences humaines et sociales.

Illich, I. (1970). Une société sans école. Paris : Seuil.

James, A. et James, A. (2004). Constructing Childhood. Theory, Policy and Social Practice. New York : Palgrave Macmillan.

Jodelet, D. (1991). Représentations sociales, dans Grand dictionnaire de la psychologie. Paris :

Larousse.

Jodelet, D. (dir.) (2003). Les représentations sociales. Paris : Presses universitaires de France.

Jutras, S., et Bisson, J. (1994). La conception de la santé chez des enfants de 5 à 12 ans. Quelques clés pour la promotion de la santé. Sciences sociales et santé, 12 (2), 5-37.

Legardez, A. et Simonneaux, L. (2006). L'école à l'épreuve de l'actualité. Enseigner les questions vives. Paris : ESF.

Lietdka, J. (2015). Perspective : Linking design thinking with innovation outcome through cognitive bias reduction. Journal of Product Innovation management, 32 (6), 925-938.

Massé, R., (2003). Éthique et santé publique. Enjeux, valeurs et normativité. Québec : Presses de l'Université Laval.

Moscovici, S., (1976). La psychanalyse, son image et son public. Paris : PUF, $2^{\mathrm{e}}$ éd.

Pereira, I. (dir.). (2019). Anthologie internationale de pédagogie critique. Paris : Éditions du Croquant.

Pransky, J. (1991). Prevention. The critical need. Springfield : Burrell Foundation.

Pruneau, D., Langis, J., Richard, J.-F., Albert, G. et Cormier, M. (2005). The evolution of children's ideas on pollution in the framework of experiential and social contructivist activities.

Environment and Sustainable Development, 4 (1), 17-34.

Pruneau, D., ElJai, B., Khattabi, A., Benbrahim, S. et Langis, J. (2017). La pensée design et Facebook au service de la résolution d'un problème d'inondation : Une étude de cas au Maroc ", Éducation relative à l'environnement, 14 (1).

Routier, C., D'Arripe, A., Soyez, S. (2017). Retour d'expérience sur une démarche de coconception, Annales des Mines - Réalités industrielles, 6-9. DOI : https://doi.org/10.3917/rindu1.172.0006

Sauvé, L. (1997), L'approche critique en éducation relative à l'environnement : origines théoriques et application à la formation des enseignants, Revue des sciences de l'éducation, 23 (1)

Sauvé, L., (2009). Vivre ensemble, sur Terre : enjeux contemporains d'une éducation relative à l'environnement, Éducation et francophonie, 37 (2). URL : https://journals.openedition.org/ere/ 2280

Scott, W. (2011). Sustainable schools and the exercising of responsible citizenship - A review essay. Environmental Education Research, 17 (3), 409-423. 


\section{NOTES}

1. Fonds de dotation rattaché aux Assurances du Crédit Mutuel (ACM).

2. Voire "politiquement vives" (Albe, 2011).

3. Notamment par le choix de thèmes d'exposés réalisés en classe ou des velléités d'adhésion à des associations de défense des animaux...

4. Qu'ils savent nommer comme telle sans que l'expression ne leur soit proposée.

5. Contrairement au G4 où les enfants se connaissaient, mais la directrice de l'école est restée dans la classe.

6. Ce qui fait partie des attentes recensées.

7. Organisation d'un atelier créatif (Poker Design) avec des adultes proches de l'univers des enfants (écrivain jeunesse, instituteur, médiateur, responsable d'association familiale, communicateur, etc.), auprès desquels les trois approches ont été testées.

\section{RÉSUMÉS}

Une enquête quantitative portant sur les attentes des enfants en matière de prévention en santé a montré que les pollutions constituent une préoccupation centrale pour cette population. Compte tenu des limites des campagnes de prévention habituelles et pour en optimiser l'efficacité, une équipe composée de deux médecins, d'une sociologue qualitativiste et d'une designer a entrepris de co-concevoir un programme de sensibilisation en y associant ses destinataires. La tranche d'âge concernée est celle des 9 à11 ans, ce qui correspond dans le système français, au dernier niveau de l'école élémentaire (CM2) et pour lequel un parcours sur l'environnement et la citoyenneté est prévu dans les programmes scolaires. Cet article a pour objectifs d'une part, de retracer l'itinéraire méthodologique sous ses différents aspects, de la préfiguration du programme à l'aboutissement d'une première proposition et d'autre part, de mettre en lumière les particularités et les exigences propres au processus de co-conception d'un tel programme. Quatre ateliers de co-conception (auprès d'enfants en territoires urbain, suburbain et rural) ont notamment rendu possible l'accès aux représentations de ceux-ci sur les différents types de pollution. Ces informations ont permis de développer un programme susceptible de fournir aux enfants les savoirs nécessaires et de les mobiliser dans l'agir pour lutter contre diverses formes de pollution. Cette démarche de co-conception, dans sa rigueur et ses exigences, poursuit des objectifs d'éducation relative à l'environnement et favorise l'écoresponsabilisation et l'autonomie des acteurs concernés.

A quantitative survey on children's expectations for health prevention has shown that they are particularly concerned about pollution. Given the limitations of the usual prevention campaigns and to maximize their effectiveness, an awareness program is co-designed with its recipients. The age group concerned is that of 9-11-year olds which corresponds, in the French system, to the last level of elementary school (CM2) and for which a course on the environment and citizenship is planned. This article aims to retrace the methodological route, from the foreshadowing of the program to the completion of a first proposal, and to analyze the requirements specific to co-construction. Four workshops were completed to access children's representations on pollution. It is about exploring the choices made the aim of producing a program that can provide children with the necessary knowledge and have an impact on their 
behaviour. The co-construction, in its rigour and requirements, reinforces the objectives of environmental education and guarantees the eco-accountability and autonomy of the players concerned.

\section{INDEX}

Mots-clés : enfant, pollution, approche participative, co-conception, prévention, santé environnementale, santé publique, éducation

Keywords : Child, pollution, participatory approach, co-construction, prevention, environmental health

\section{AUTEUR}

\section{CLARA BOUTET}

Clara Boutet est ethno-anthropologue de formation. Elle suit l'élaboration du programme de coconstruction dont il est ici question pour en décrire la méthodologie, de sa conception à sa diffusion. Elle entreprend d'intégrer ce travail dans un processus de recherche traitant le principe de co-construction en prévention santé, par la réalisation d'une thèse de doctorat en anthropologie sociale (Université de Strasbourg, UMR 7367). 